\title{
Far-field Subdiffraction Imaging of Semiconductors Using Nonlinear Transient
}

\section{Absorption Differential Microscopy}

\author{
Ning Liu ${ }^{\dagger,}$, Mahendar Kumbham ${ }^{\dagger,}$, Isabel Pita ${ }^{\dagger,}$, Yina Guo ${ }^{\ddagger}$, Paolo Bianchini ${ }^{\S}$, Alberto \\ Diaspro $^{\S, \|}$, Syed A. M. Tofail ${ }^{\dagger, \downarrow}$, André Peremans ${ }^{\perp}$, Christophe Silien ${ }^{\dagger, t, *}$ \\ †Department of Physics and Energy, University of Limerick, Ireland \\ ॠMaterials and Surface Science Institute, University of Limerick, Ireland \\ ${ }^{\S}$ Department of Nanophysics, Istituto Italiano di Tecnologia, Genoa, Italy

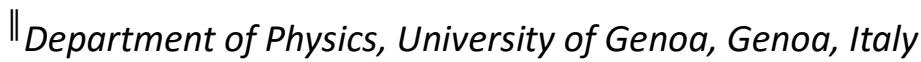 \\ ${ }^{\perp}$ Centre de Recherches en Physique de la Matière et du Rayonnement, Université de Namur, \\ Namur, Belgium
}

* To whom correspondence should be addressed. E-mail: christophe.silien@ul.ie

S1. Matlab simulations of TA and DTA imaging with non-linearity in the transient absorption.

The spatial resolution in TA and DTA microscopy described in the main text results from the non-linearity of the TA signal as a function of the pump fluency and from the differentiation with a transient signal generated with a pump of different geometry and/or power. Key to this paper is that saturation in the TA is not required and that substantial improvement in spatial resolution with respect to the diffraction limit is possible at pump fluency that does not largely exceed the non-linear transition threshold $\left(p_{0}\right)$. In the experiments, the spatial definition of the differentiation pump is that of a so-called doughnut beam generated using a vortex phase plate (0 to $2 \pi$ ). In the case of semiconductor nanostructures such as the CdSe nanobelts, the non-linearity corresponds to a reversal of the TA signal slope and sign versus the pump power above a threshold $p_{0}$ (see Fig. 2 in the main text for experimental measurements). For CdSe the non-linearity stems from the competing influence of interband absorption bleaching and induced intraband free carrier absorption (see main text for details). The non-linearity observed is common to many semiconductors.

The differential scheme leads to an increase in lateral resolution at fluency below the non-linearity threshold by exploiting a doughnut intensity pattern. The resolution is then critically dependent on the quality of the doughnut pump. For CdSe nanobelts and other materials exhibiting similar non-linearity in the TA signal, an increase in the spatial resolution is also achieved with a Gaussian pump, at pump fluency just above the said threshold. Indeed with a Gaussian pump the TA signal slope reversal can be localized to a radius smaller than the probe beam (see also Fig. 1 in the main text). A priori, any change of slope in 
the TA signal (including TA saturation) will lead to a similar effect, yet the slope reversal is critical for the effect to be exploitable at reasonable signal-to-noise ratio. The differentiation with another pump intensity profile and/or power is not required for introducing longer spatial frequencies but it allows for reducing the influence of the shorter spatial frequencies on the image.

TA and DTA images were simulated using Matlab to exemplify the discussion above. In a coherent scanning imaging system, the image formation with the beams focused in the sample plane can be approximated by

$$
I_{0}(x, y)=\left|a_{0}(x, y)\right|^{2}=\left|\iint_{-\infty}^{+\infty} P S F_{0}\left(x^{\prime}, y^{\prime}\right) \times o\left(x-x^{\prime}, y-y^{\prime}\right) d x^{\prime} d y^{\prime}\right|^{2},
$$

where $\operatorname{PSF}_{0}(x, y)$ is the amplitude point-spread function, $a_{0}(x, y)$ is the amplitude image and $I_{0}(x, y)$ is the intensity image. The object function $o(x, y)$ is written as a black and white function, equals to 1 where the nanobelt is found and zero elsewhere. When the sample is first excited by the pump beam, the image may thus be written as

$$
I_{p}(x, y)=\left|a_{p}(x, y)\right|^{2}=\left|\iint_{-\infty}^{+\infty} P S F_{p}\left(x^{\prime}, y^{\prime}\right) \times o\left(x-x^{\prime}, y-y^{\prime}\right) d x^{\prime} d y^{\prime}\right|^{2},
$$

where

$$
P S F_{p}(x, y)=P S F_{0}(x, y) \times(1-e[p(x, y)])
$$

and $e[p(x, y)]$ is a function of the pump intensity and represents the change in reflectivity of the object following optical pumping. In the experiments the TA images corresponds to the difference between the images recorded with and without pump,

$$
I_{T A}(x, y)=I_{0}(x, y)-I_{p}(x, y) .
$$

For DTA, the images are the difference between two TA images with different pump. For simplicity, $\operatorname{PSF}_{0}(x, y)$ is written as a Gaussian field function (LGO0) of waist adjusted to reproduce the fwhm of 380-400 $\mathrm{nm}$ measured in the images of small-object in the experiment at $\lambda=678 \mathrm{~nm}$. The pump field is described by a Gaussian field function (LGOO) or a Laguerre-Gaussian field function (LG01) of same waist. The change in reflectivity $e$ was assumed to be linear with a negative slope below a set intensity threshold to reach of loss of $10 \%$ at the threshold, and linear with a positive slope beyond. The slope ratio was adjusted to best reproduce the experimental plot of TA versus pump power measured the center of a $250 \mathrm{~nm}$ wide nanobelt (see Fig. S1 (a) below and Fig. 2 in the main text).

TA and DTA intensity image profiles for a thin nanobelt with the pumps below and above the non-linearity threshold $p_{0}$ are shown in Fig. S1 (b) and (c). The plots are reminiscent of the experimental observations reported in the main text in Fig. 2). The full-width at half maximum of the DTA profile, with the baseline taken at the minimum, is ca. $220 \mathrm{~nm}$ when the pump is kept below the threshold, and results from the node dimensions in the doughnut pump. The width of the dip in the TA profile with the pump above the threshold is ca. $100 \mathrm{~nm}$. The latter value varies with the Gaussian pump power and is not 
significantly changed in the DTA profile. Moreover, the second pump does not have to be doughnut and better subtraction can be achieved using for example a second Gaussian pump (see below).

The effective amplitude $P S F_{p}$ is plotted in Fig. S1 (d) for different Gaussian pump power and is compared to $P S F_{0}$. Beyond the non-linearity threshold $p_{0}$, the dip in $P S F_{p}$ deepens and widens when the pump power is increased. The dip is associated with new spatial frequencies (Fig. S1 (e)) beyond those defined by the diffraction-limited $P S F_{0}$ and is the source of super-resolution in TA and DTA. With respect to TA, the difference with a reference pump in DTA aims at further emphasizing these new longer spatial frequencies and relatively decreasing the influence of the shorter ones, for a better visualization of the otherwise unresolved features in the images. For comparison, the effective amplitude $P S F_{p}$ for different doughnut pump power and assuming that the change in reflectivity $e$ saturates beyond the non-linearity threshold are also shown (see Fig. S1 (f) and (g)).
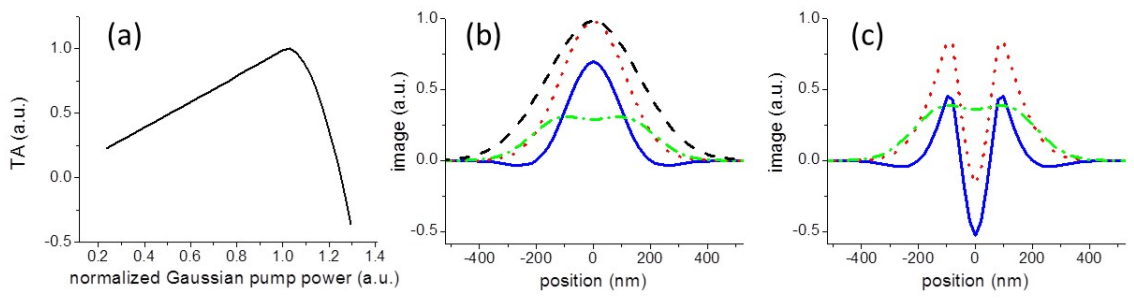

(d)

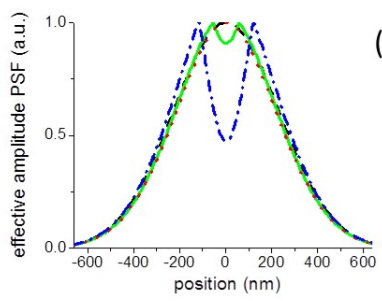

(e)

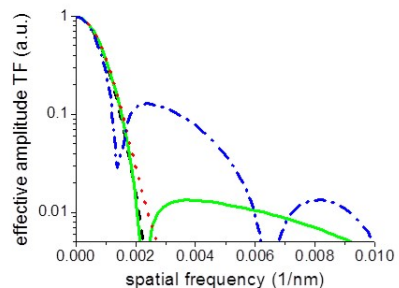

(f)

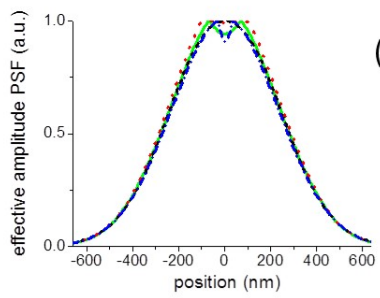

(g)

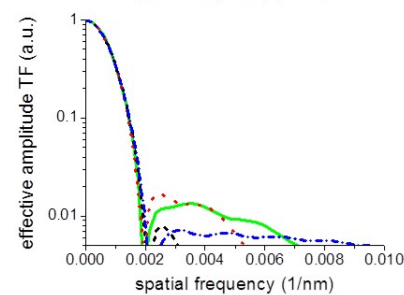

Fig. S1: Simulations of TA and DTA profiles and PSFs. (a) Computed TA intensity at the center of a $250 \mathrm{~nm}$ wide CdSe nanobelt as a function of the Gaussian pump power normalized to the non-linearity threshold $p_{0}$. The TA intensity is set to 1 at maximum value in the plot. (b) Computed intensity imaged across a 20 $\mathrm{nm}$ wide CdSe nanobelt (black/dash: in the absence of pump, intensity maximum set to 1; red/dot: TA with Gaussian pump at $0.95 p_{0}$, intensity maximum set to 1; green/dash-dot: idem with doughnut pump at $0.85 p_{0}$, intensity normalized the same as for Gaussian-pumped TA; blue/solid: DTA with Gaussian and doughnut pumps at 0.9 and $0.85 p_{0}$ ). (c) Same as (b) with Gaussian and doughnut pumps at 1.2 and 0.95 $p_{0}$. (d) Effective amplitude PSF (PSFp) for CdSe normalized to 1 (black/dash: in the absence of pump; red/dot: with Gaussian pump at $0.95 p_{0}$; green/solid: with Gaussian pump at $1.1 p_{0}$; blue/dash-dot: with Gaussian pump at $1.3 p_{0}$ ). (e) Effective amplitude transfer function for same parameters as in (d). (f) Effective amplitude PSF (PSFp) for a doughnut pump with $e(p)$ saturating beyond $p_{0}$ (black/dash: at $1.2 p_{0}$; red/dot: at $5.9 p_{0}$; green/solid: at $12 p_{0}$; blue/dash-dot: at $59 p_{0}$ ). (g) Effective amplitude transfer function for same parameters as in (f). 
Simulated images of a pair of $10 \mathrm{~nm}$ wide, 1 micron long nanobelt are shown in Fig. S2. When separated by $300 \mathrm{~nm}$, the nanobelts can be resolved by DTA at power below the non-linearity threshold when using a doughnut pump for the differentiation, while the nanobelts are not resolved in the TA and $I_{0}(x, y)$ images (Fig. S2 (a-c)). When the nanobelts are closer and separated by $150 \mathrm{~nm}$, resolving the nanobelts requires setting the Gaussian pump above the said threshold. DTA with a second Gaussian pump set below the threshold provides then a good subtraction (Fig. S2 (d)). Remarkably, the ability to further resolve adjacent nanobelts is strongest when the pump power matches with the threshold, and it is gradually decreasing when the power increases. This contrasts with the RESOLFT-based approaches, where the power-induced shrinking of a doughnut intensity profile is used to enhance the spatial resolution. Experimentally, the resolution is thus here primarily limited by the signal-to-noise ratio. Would the specimen TA exhibit a saturation and be constant beyond the threshold, the new spatial frequencies accessible by setting the Gaussian pump just above the threshold are weaker, making DTA with a Gaussian pump subtraction of limited practicality (Fig. S2 (e)). A RESOLFT-based DTA scheme with a doughnut-shaped subtraction pump is then advantageous. With the optical scheme unchanged, other than the specimen showing a saturation instead of the slope reversal, it is shown that resolving the nanobelts apart by $150 \mathrm{~nm}$ requires a doughnut power ca. 4.4 times above the threshold (Fig. S2 (f)).
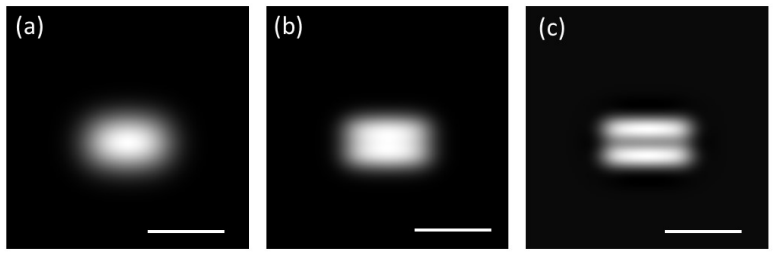

(d)
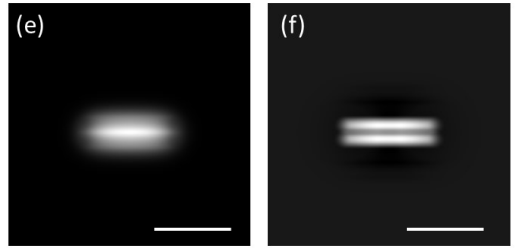

Fig. S2: Simulations of TA and DTA images of two nanobelts. (a) Computed image of 2 CdSe nanobelts $\left(10 \times 1000 \mathrm{~nm}^{2}\right)$ apart by $300 \mathrm{~nm}$ in the absence of pump. (b) TA image of the same nanobelts with a Gaussian pump at $0.95 p_{0}$. (c) DTA image of the same nanobelts with a Gaussian pump at $0.95 p_{0}$ and doughnut pump at $0.85 p_{0}$. (d) DTA image of 2 CdSe nanobelts $\left(10 \times 1000 \mathrm{~nm}^{2}\right)$ apart by $150 \mathrm{~nm}$ with two Gaussian pumps at 1.2 and $0.95 p_{0}$. (e) idem with $e(p)$ saturating beyond $p_{0}$. (f) idem with $e(p)$ saturating beyond $p_{0}$ and Gaussian and doughnut pumps at 11 and $4.4 p_{0}$. All images are in linear grey scale with higher intensities white. Scale bars $1000 \mathrm{~nm}$.

\section{S2. Phase sensitive heterodyne detection (double chopping detection).}

Because part of the pump beam intensity reaches the detector, a double chopping scheme was implemented to extract directly the transient absorption signal, which is the probe intensity variation resulting from irradiation with the pumps. The detection scheme involves chopping the Gaussian and 
second (usually a doughnut) pumps in alternation at $500 \mathrm{~Hz}$ and chopping the probe at $333 \mathrm{~Hz}$. The detector signal is then measured by a lockin amplifier synchronized at $167 \mathrm{~Hz}$. In the experiment all these frequencies are synchronized to the laser pulse rate of $1 \mathrm{kHz}$. Fig. S3 (a) and (b) represent the intensity pulses measured by the detector by sequential opening of the Gaussian and doughnut beams and by the probe, respectively. The intensity pulses are simulated by Gaussian functions for simplicity. The simulation considers that the measured pump and probe intensities are of same magnitude at the detector. These time traces were created with MATLAB. The total signal at the detector when the Gaussian pump (blue) causes a bleaching of the absorption but not the doughnut (orange) was then computed as the sum of the Gaussian, doughnut, and probe (red) pulse series to which 10 percent of the product between the Gaussian and probe pulses series was added to simulate the probe intensity increase. When none of the pumps induce bleaching, the signal at the detector is computed as the sum of the Gaussian, doughnut, and probe only. The resulting curves are shown in Fig. S3 (c) and (d), respectively. An oscillation of $6 \mathrm{~ms}$ period can be observed on the first curve. Moreover, as shown by the computed fast Fourier transform of the two previous curves displayed in Fig. S3 (e) and (f), the interaction between pump and probe results in the apparition of frequencies at 167 and $833 \mathrm{~Hz}$. These frequency components are not seen in the absence of interaction. The lockin amplifier effectively measures the Fourier component of the signal at the detector thus extracting the transient absorption.
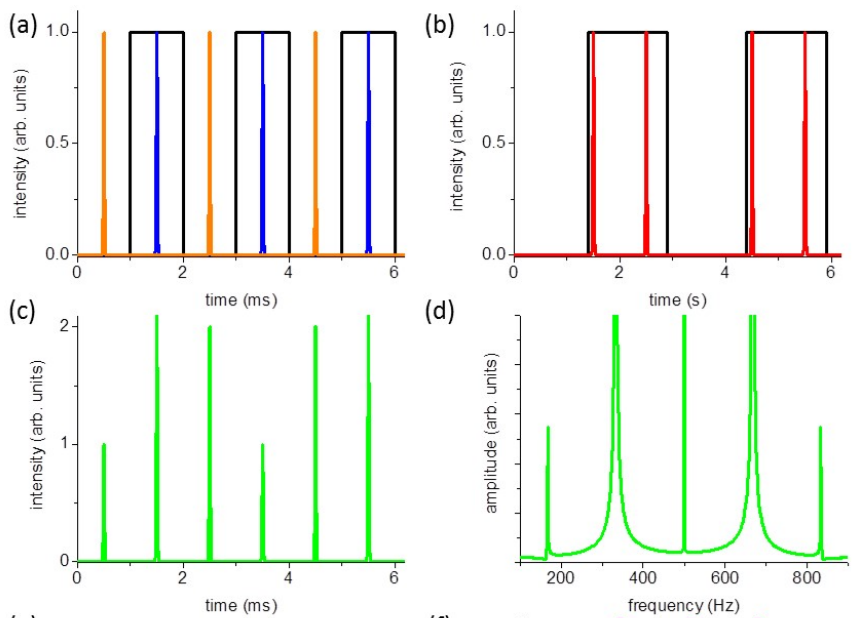

(d)
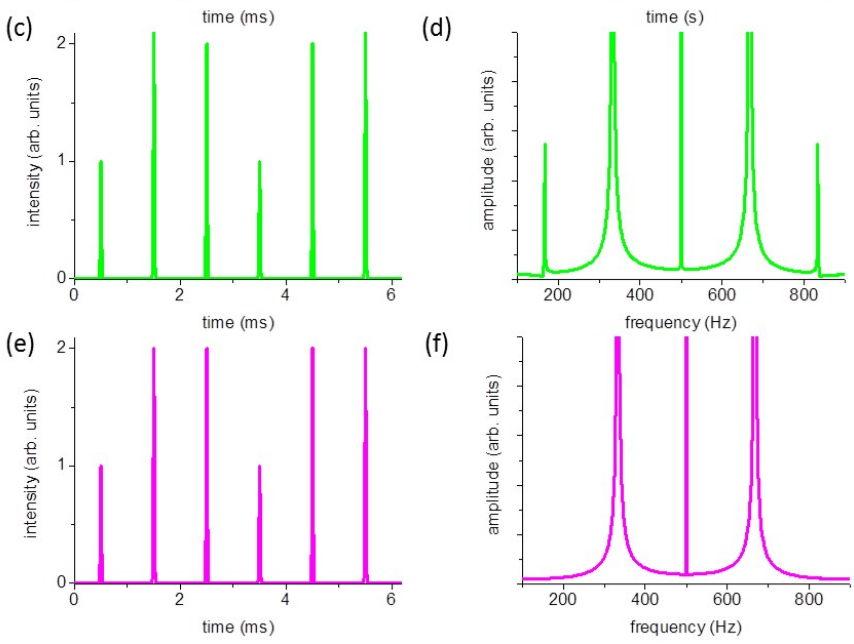

Fig. S3. Phase sensitive heterodyne detection in TA and DTA. Simulated pump signal at the detector following Gaussian and doughnut pump illumination (a), probe illumination (b), total pump and probe illumination in the presence of bleaching by the Gaussian pump (c), in the absence of bleaching (d). The fast Fourier transforms of the curves in (c) and (d) are shown respectively in (e) and (f). 


\section{S3. COMSOL model geometry for simulating nanobelt line profile in backscattered images.}

The Wave Optics module in COMSOL MULTIPHYSICS 4.4 simulation package was used to simulate image line profiles of CdSe nanobelts. A 3D model was used in order to model properly the polarization effect of the reflected/scattered fields. A 3D Gaussian beam (beam waist $260 \mathrm{~nm}$ ) was applied as background field to simulate the excitation beam and the frequency domain stationary solutions were obtained for a series of simulation parameter sets. In order to reduce the calculation time and yet still capture the essential physics, the simulations were run in a spherical domain with scattering boundary condition and without perfect matching layers. Fig. S4 (a) shows the mode geometry. To simulate the total power received by the photodetector, we defined a spherical shell in the far-field domain with the apex angle of the solid angle determined by the numerical aperture of the objective $(N A=0.9)$. From $N A=n \sin (\theta)$, the apex angle $2 \theta$ is $128.3^{\circ}$. The thickness of the shell was chosen to be half of the wavelength to account for interferences. The total power received by the photodetector was assumed proportional to the integral of the electric field intensity over the volume of shell. The magnetic field detected by the photodetector was supposed negligible.

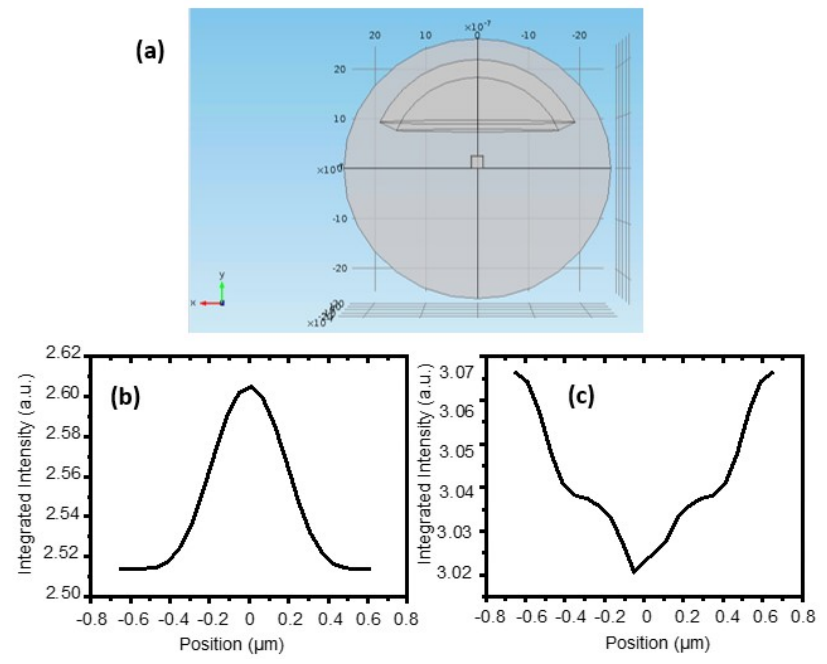

Fig. S4: COMSOL model geometry. (a) CdSe nanobelts are set to be $3.1 \mu \mathrm{m}$ long with their long axis is aligned with the $z$ direction. (b) Line profiles for a nanobelt of width $200 \mathrm{~nm}$ and thickness $140 \mathrm{~nm}$. (c) Line profile for a nanobelt of width $250 \mathrm{~nm}$ and thickness of $240 \mathrm{~nm}$.

Reflected and scattered beams by the glass substrate $(n=1.52)$ and the CdSe nanobelts $(n=2.76072$, $k=0.24217$ ) were computed directly from the scattered field $E_{s}$. To simulate the scanning of the sample in the beam, the position of the nanobelt was varied along the $x$ axis, with the other parameters unchanged. The imaged line profiles in reflective mode (backscattered) were then approximated by the integrated intensity of the electric field over the far-field spherical shell as a function of the position of the nanobelt along the $x$ axis. Two examples of simulated line profiles are shown in Fig. S4 (b) and S4 (c). Backscattering occurs over both substrate and nanobelt, and a nanobelt may be imaged as a protrusion (see for example NB1 in Fig. 4a and NB2 in Fig. 5a) or as a depression (see for example NB5 in Fig. S8(a) and NB6 in Fig. S9 (b)). Finite element simulations of the backscattered line profiles (Fig. S4(c)) confirm that the contrast in the backscattered images involves interferences between light backscattered by the nanobelt and reflected by the glass substrate. Depending on the nanobelt thickness, these interferences may be destructive, leading to the nanobelt being imaged as depression. 


\section{S4. Method for estimating the nanobelt width and thickness by matching experimental backscattered images to simulated COMSOL line profiles.}

The width of the CdSe nanobelts (NB1 and NB3) presented in the manuscript cannot be directly derived from the diffraction limited backscattered images. To assess the physical width of these nanobelts, in particular those that were not imaged by SEM after optical TA/DTA microscopy, we matched their experimental backscattered images to simulated COMSOL line profile, computed for a series of nanobelt of various width and thickness (see Fig. S5), using the model presented in the Supplementary Information section S3. We also assumed (i) that the thickness of the nanobelt is not larger than their width (in other words, we assumed that the nanobelts remain flat on their larger face when on the glass surface, which is a reasonable assumption from the experience of working with similar nanobelts) and (ii) that the physical width of the nanobelt is not larger than the FWHM measured in the DTA image. The physical width, thickness, and their standard deviation are thus estimated from the plot in Fig. S5, by matching the experimental FWHM to the simulated plots while taking into account of (i) and (ii). For example, for NB1 (see main text Fig. 4) the backscattered and DTA images reveal FWHMs of $483 \pm 10 \mathrm{~nm}$ and $269 \pm 10 \mathrm{~nm}$, respectively. The physical width of NB1 is thus estimated to lie between 180 and $269 \mathrm{~nm}$, and we conclude that the width and height are respectively $220 \pm 50 \mathrm{~nm}$ and $150 \pm 15 \mathrm{~nm}$. For NB3 (see main text Fig. 5), we conclude that the width and height are respectively $280 \pm 50 \mathrm{~nm}$ and $150 \pm 15 \mathrm{~nm}$.

The COMSOL-based approach was further validated by SEM images of NB2 and NB4 (see Fig. 5), which reveal physical width of $82 \pm 3$ and $56 \pm 3 \mathrm{~nm}$, respectively. Using a physical width of $82 \mathrm{~nm}$, the COMSOL simulations show that the backscattered line profile width has very little dependence on the nanobelt height and that the FWHM is expected at ca. $382 \mathrm{~nm}$. This number relates well to the experimental value of $392 \pm 10 \mathrm{~nm}$ and is about $3 \%$ less. For a physical width of $56 \mathrm{~nm}$, the COMSOL simulations show a backscattered FWHM value of $362 \mathrm{~nm}, 12 \%$ less than the measured FWHM $(412 \pm 10$ $\mathrm{nm}$ ). Fig. S9 shows that the physical dimension of the NB6 is $309 \pm 3 \mathrm{~nm}$ (measured by SEM). In the backscattered image, NB6 appears as a depression of FWHM $808 \mathrm{~nm}$. The COMSOL scheme suggests a physical dimension of ca. $260 \mathrm{~nm}$, which is $16 \%$ less than the SEM-measured physical width. The COMSOL model obviously simplifies the imaging process, the simulation size, which is limited by the memory of the computer) and further assumes ideal focus and light collection. Yet, in keeping with their accuracy detailed above, the estimations allow us to shed further light on the TA and DTA data for these nanobelts that were not imaged by SEM.
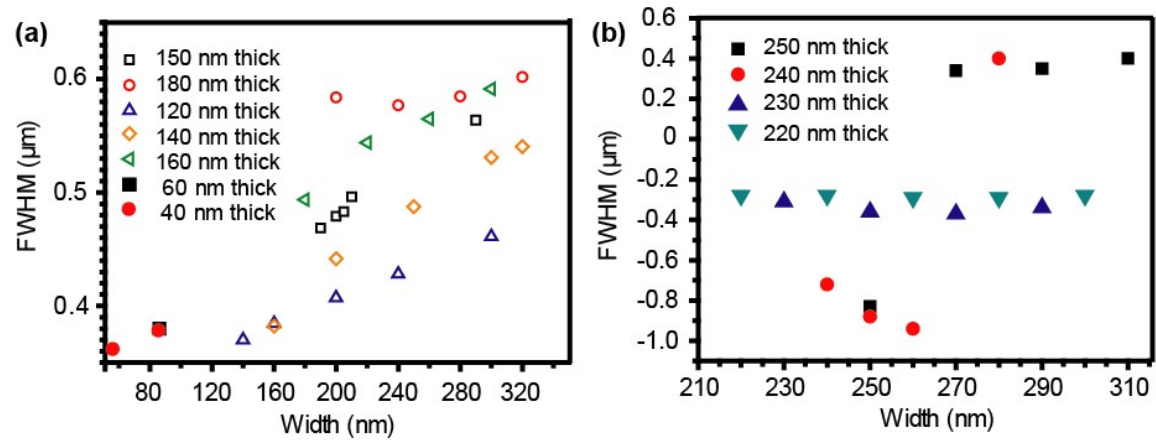

Fig. S5: Simulated COMSOL FWHM. COMSOL-simulated backscattered FWHM for CdSe nanobelts of various width and thickness. (a) Computed backscattered FWHMs for NBs of thickness ranging from 40 $\mathrm{nm}$ to $180 \mathrm{~nm}$ with the line profiles showing all protrusions. (b) Computed backscattered FWHMs for NBs ranging from $220 \mathrm{~nm}$ to $250 \mathrm{~nm}$. Line profile appearing as depression are indicated as negative FWHM values. 


\section{S5. Nanobelt backscattered image width with polarized detection.}

Because the TA is achieved with the pump and probe beams at same wavelength, we have introduced a polarization scheme that minimizes the pump intake by the photodetector (see Methods and Fig. 1b). Linear polarization of the pump beam was achieved after WP3 and only ca. 5\% of the pump power remains after LP to be collected by the photodetector. The double chopping scheme is then sufficient to isolate the TA signal at $167 \mathrm{~Hz}$ from the reflected probe power. The probe beam remains however elliptical after WP3 so that only ca. $60 \%$ of the probe power is collected by the photodetector.
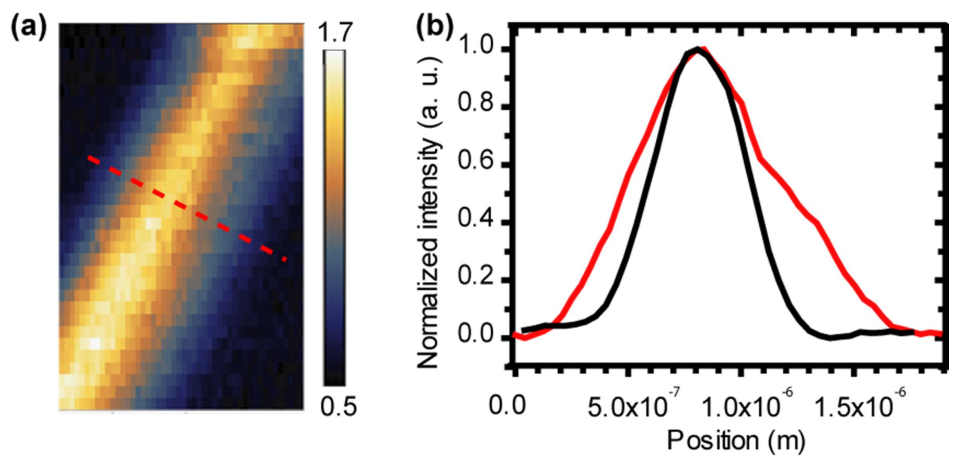

Fig. S6: Nanobelt backscattered image width and polarized detection. (a) Backscattered probe image of NB1 with WP3 and LP in place. (b) Line profiles across NB1 without WP3/LP (black, extracted from Fig. 4a and with WP3/LP (red, extracted from Fig. S6(a)). The curves were normalized to a maximum of 1.

By comparison to the backscattered probe image of NB1 shown in Fig. 4a and recorded without the combination WP3/LP, Fig. S6 reveals that when WP3 and LP are in place the apparent width of the nanobelt is increased. In Fig. S6, the FWHM is $733 \pm 10 \mathrm{~nm}$ while $483 \mathrm{~nm}$ was measured in Fig. 4a. The contribution to the TA image of each polarization component in the probe is thus not measured uniformly and this leads to a variation in the propagating fields (from CdSe and glass) reaching the detector and eventually a variation in the measured intensity, with an apparent broadening of the optical backscattered and TA images when WP3/LP are in place. However, in the DTA image the effect of both pumps is similar away from the object (because in a differential scheme the subtracted images are weighted to achieve the best suppression of the signals away from the object and this is done here by adjusting the relative energy of the two pumps before scanning as the differentiation is directly performed by the lockin amplifier) and thus the DTA images highlight principally the changes in the probe image due to the pump intensity profile on top of the nanobelt.

The effect of WP3/LP was simulated by COMSOL by adapting the model presented in the Supplementary Information S3. The probe beam after WP3 can be approximated by:

$$
\begin{gathered}
E_{p x}=\sin \left(\theta-\frac{\pi}{4}\right)\left[E_{s x} \sin \left(\theta-\frac{\pi}{4}\right)+E_{s z} \cos \left(\theta-\frac{\pi}{4}\right)\right]\left[e^{j \pi / 2}-1\right]+E_{s x} \\
\text { and } E_{p z}=\cos \left(\theta-\frac{\pi}{4}\right)\left[E_{s x} \sin \left(\theta-\frac{\pi}{4}\right)+E_{S z} \cos \left(\theta-\frac{\pi}{4}\right)\right]\left[e^{j \pi / 2}-1\right]+E_{S z}
\end{gathered}
$$


where $E_{p x}$ and $E_{p z}$ are the scattered field components after WP3 in the $x$ and $z$ directions respectively, where $E_{S x}$ and $E_{S Z}$ are the scattered fields components before WP3 in the $x$ and $z$ directions respectively, and where $\theta$ is the polarization angle of the probe beam with respect to $z$ direction (see Fig. S4(a)). After the linear polarizer LP, the intensity collected by the detector is then given by:

$$
I_{L P}=\left|\sin (\alpha) E_{p x}+\cos (\alpha) E_{p z}\right|^{2}
$$

where $\alpha$ is the angle between the direction of polarization of LP and the $z$ axis.

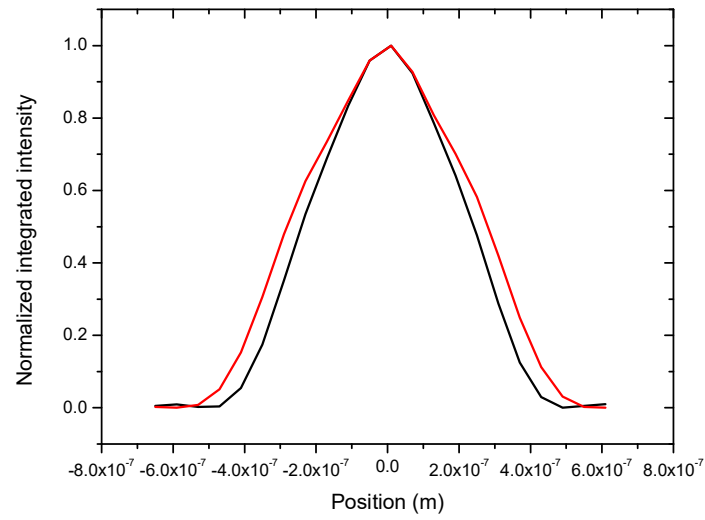

Fig. S7: COMSOL simulation and polarized detection. Simulated backscattered image profile across a CdSe nanobelt (thickness=205 nm; height=150 nm) with WP3/LP (red) and without WP3/LP (black).

The backscattered image profiles across the nanobelts were constructed by integrating $I_{L P}$ over the far field spherical shell representing the detector whilst varying the position of the nanobelt along $x$ axis (see Supplementary Information S3). In line with the experimental observations reported in Fig. S6, the simulated line profile with WP3/LP is seen wider than without (568 nm and $483 \mathrm{~nm}$ respectively, see Fig. S7). We note that, although the FWHM increase is observed, its magnitude is less than seen in the experiment, which we attribute to the simplicity of the model, especially in the definition of role played by WP3.

\section{S6. Supplementary TA/DTA data.}

As the focused beam diameter is larger than most of our CdSe nanobelts, backscattering includes interference of light reflected from both glass substrate and NB, and it results that the NB can be imaged as a protrusion (see NB1, NB2, NB3, and NB4 in the main text) or as depression (see NB5 in Fig. S8(a) and NB6 in Fig. S9) in the backscattered image, depending on their physical width and height. COMSOL simulations (see Supplementary Information S3) confirm these observations. TA/DTA microscopy, on the other hand, is sensitive to the optical absorption of the semiconductor materials and the DTA amplitude is zero away from the nanobelts. The NBs are thus, in the bleaching regime, below the non-linearity, systematically imaged as protrusions with sub-wavelength resolution whether their backscattering image shows a protrusion (see NB1 and NB2 in the main text and NB3 in Fig. S8(b)) or a depression (see below, Fig. S8(a) and NB6 in Fig. S9). Conversely, the NBs are systematically imaged as depressions by TA/DTA in the induced absorption regime (see NB3 and NB4 in the main text). 

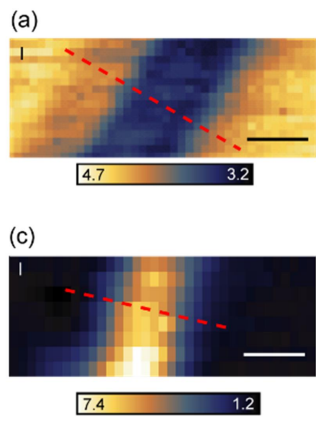
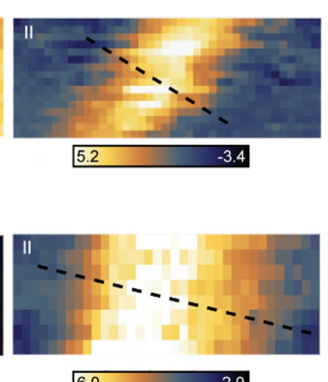

6.0
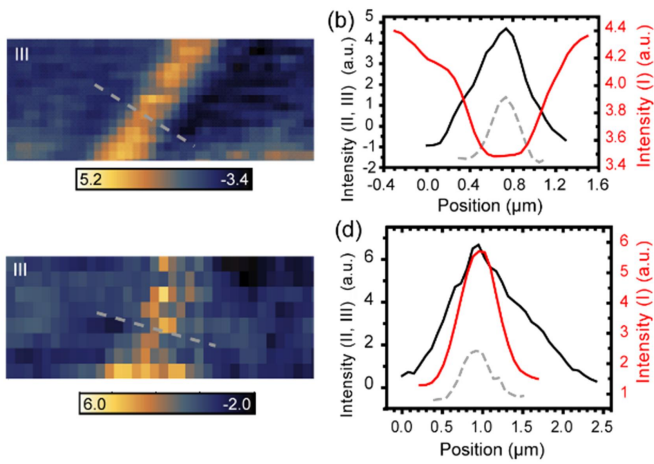

Fig. S8: TA/DTA of other nanobelts. (a) Backscattered, TA and DTA images of NB5 (Gaussian pump = $59 \mathrm{nW}$; doughnut pump $=60 \mathrm{nW}$; probe $=12 \mathrm{nW}$ ). Scale bar $500 \mathrm{~nm}$. (b) Line profiles extracted from the dashed lines indicated in (a). The values of FWHM are also listed in table S1. (c) Backscattered, TA and DTA images of NB3 at low pump intensity (Gaussian pump $=44 \mathrm{nW}$; doughnut pump $=56 \mathrm{nW}$; probe $=12 \mathrm{nW}$ ). The DTA image was computed as the difference between two TA images recorded with Gaussian and doughnut pumps and is thus of lower signal-to-noise ratio than those recorded with the two pumps in alternation using the lockin. (d) Line profiles extracted from the dashed lines indicated in (c). The values of FWHM are also listed in table S1.

In DTA the FWHM for thin NBs is expected to be at least $220 \mathrm{~nm}$ in the linear regime (see Supplementary Information S1). For NBs that are much wider than this value, the FWHM in DTA is expected to be very close to the NB physical width. This has been verified for NB6 which has a physical width of $309 \pm 3 \mathrm{~nm}$ (as measured by SEM) and which shows a FWHM of $319 \pm 10 \mathrm{~nm}$ in the DTA image recorded with a doughnut and below the non-linearity threshold.
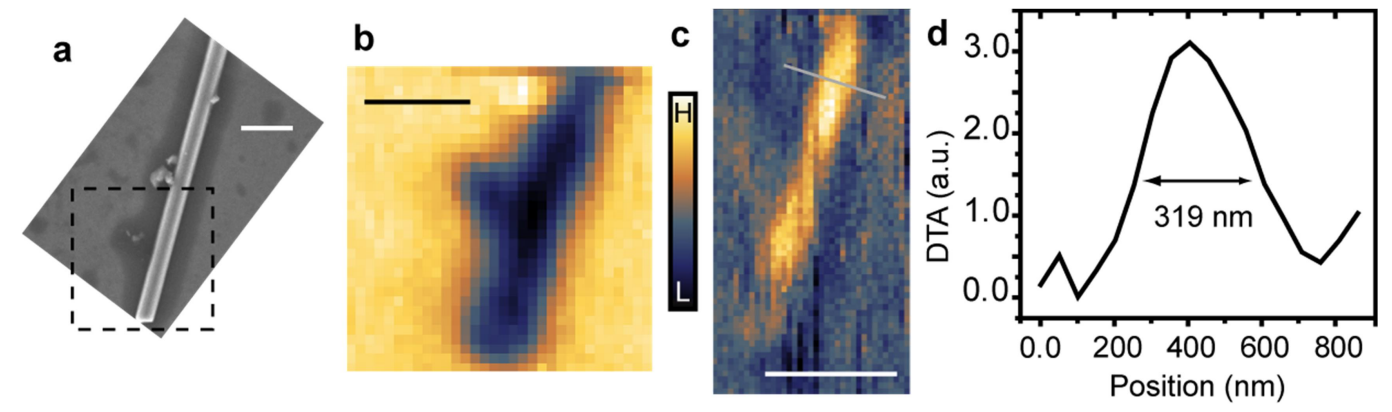

Fig. S9: SEM and DTA of a wide nanobelt. (a) SEM image of NB6. (b) Backscattered image of NB6 from the area highlighted by the dash square in (a). (c) DTA image of NB6 with Gaussian pump $66 \mathrm{nW}$, doughnut pump $76 \mathrm{nW}$, and probe $11 \mathrm{nW}$. All scale bars are $1 \mu \mathrm{m}$. (d) Line profile extracted from (c).

In table 1 we summarize the physical dimensions of the nanobelts presented in the main text and here in S6, along with the various width measured in the optical images. 
Table S1: NB physical and optical dimensions. Comparison of the backscattered, TA, and DTA image half-widths with the estimated (e) with COMSOL (see Supplementary Information S3)/measured (m) by SEM nanobelt physical widths. I and $\mathrm{nl}$ indicate whether the Gaussian pump power was used in the linear or non-linear regime, respectively. $d$ refers to the backscattered image showing the nanobelt as a depression.

\begin{tabular}{|l|l|l|l|l|l|l|}
\hline NA0.9(679nm) & NB1 (Fig. 4a) & NB2 (Fig. 5a) & NB3 (Fig. 5b) & NB4 (Fig. 5e) & NB5 (Fig. S8) & NB6 (Fig. S9) \\
\hline Physical width & $220 \pm 50 \mathrm{~nm}(\mathrm{e})$ & $82 \pm 3 \mathrm{~nm}(\mathrm{~m})$ & $280 \pm 50(\mathrm{e})$ & $56 \pm 3 \mathrm{~nm}(\mathrm{~m})$ & $270 \pm 50 \mathrm{~nm}(\mathrm{e})$ & $309 \pm 3 \mathrm{~nm}(\mathrm{~m})$ \\
\hline Backscattered & $483 \pm 10 \mathrm{~nm}$ & $392 \pm 10 \mathrm{~nm}$ & $547 \pm 10 \mathrm{~nm}$ & $412 \pm 10 \mathrm{~nm}$ & $791 \pm 10 \mathrm{~nm}(\mathrm{~d})$ & $808 \pm 10 \mathrm{~nm}(\mathrm{~d})$ \\
\hline TA & $847 \pm 10 \mathrm{~nm}$ & $450 \pm 10 \mathrm{~nm}$ & $990 \pm 10 \mathrm{~nm}$ & $525 \pm 10 \mathrm{~nm}$ & $520 \pm 10 \mathrm{~nm}$ & $325 \pm 10 \mathrm{~nm}$ \\
\hline DTA & $269 \pm 10 \mathrm{~nm}(\mathrm{l})$ & $248 \pm 10 \mathrm{~nm}(\mathrm{l})$ & $330 \pm 10 \mathrm{~nm}(\mathrm{l})$ & & $295 \pm 10 \mathrm{~nm}(\mathrm{l})$ & $319 \pm 10 \mathrm{~nm}(\mathrm{I})$ \\
& & & $313 \pm 10 \mathrm{~nm}(\mathrm{nl})$ & $185 \pm 10 \mathrm{~nm}(\mathrm{nl})$ & & \\
\hline
\end{tabular}

\section{S7. TA/DTA sensitivity.}

The sensitivity of the TA/DTA experiments is here primarily limited by the laser and OPA pointing (mrad) and power (3\%) instabilities, as well as by the small temporal jittering which further affects the synchronous detection with the double chopping scheme given the laser pulse rate of $1 \mathrm{kHz}$.

The TA measurable with our system is first determined from the data recorded on NB1 (see Fig. 4, estimated physical width $220 \pm 50 \mathrm{~nm}$ ). When the polarization scheme is in place, the backscattered image of the probe (power $7 \mathrm{nW}$ ) measured at $333 \mathrm{~Hz}$ in the absence of pump shows a maximum signal of $1.55 \pm 0.05 \mathrm{~V}$ (Fig. S6(a), lockin sensitivity $200 \mu \mathrm{V}$, time constant $50 \mathrm{~ms}$ ) across the belt. With the Gaussian pump (power $53 \mathrm{nW}$ ), doughnut pump $(65 \mathrm{nW})$ and the lockin set at $166.7 \mathrm{~Hz}$, the maximum DTA signal across NB1 is $0.20 \pm 0.04 \mathrm{~V}$ (lockin sensitivity $20 \mu \mathrm{V}$, time constant $500 \mathrm{~ms}$ ). Thus, the change of reflectance over the total reflectance $\Delta R / R$ revealed in the DTA is ca. $1.3 \pm 0.3 \%$. For NB4, the thinnest belt measured in our experiments (physical width $56 \pm 3 \mathrm{~nm}$ ), we obtain that $\Delta R / R$ is ca. $1.9 \pm 0.6 \%$ (lockin sensitivity $10 \mu \mathrm{V}$, time constant $1 \mathrm{~s}$, Gaussian pump $76 \mathrm{nW}$, probe $9 \mathrm{nW}$ ). With the TA signal-to-noise ratio of ca. 3:1 NB4 is among the thinnest CdSe nanobelts that can be investigated with our experimental setup.

Extrapolating to a single CdSe nanoparticle of diameter $20 \mathrm{~nm}$ and assuming that the reflectance of the latter is proportional to its cross-section area and that the absorbance is unchanged, it is deduced that a ca. 8 times increase $\left(56^{2} / 20^{2}\right)$ in sensitivity is required with respect to our TA system. These performances are however achievable with other TA microscopy systems demonstrated in the literature. For example, with repetition rates $>5 \mathrm{MHz}$, TA microscopy on $20 \mathrm{~nm}$ Au particles and CdSe nanowires has been demonstrated with signal-to-noise ratio ca. 120:1 [Lo, S. S.; Devadas, M. S.; Mahor, T. A.; Hartland G. V. Optical detection of single nano-objects by transient absorption microscopy Analyst 138, 25 (2013)]. Moreover, in ref. 1, graphene platelets of average diameter $100 \mathrm{~nm}$ have been measured at similarly high signal-to-noise ratio with very short dwell time $<5 \mu$ s and a laser pulse rate of $80 \mathrm{MHz}$. 